\title{
Stromal Expression of MicroRNA-21 in Advanced Colorectal Cancer Patients with Distant Metastases
}

Kyu Sang Lee ${ }^{1}$. Soo Kyung Nam ${ }^{1}$ Jiwon Koh' ${ }^{2}$. Duck-Woo Kim ${ }^{3}$ Sung-Bum Kang ${ }^{3}$. Gheeyoung Choe ${ }^{1,2}$ Woo Ho Kim ${ }^{2}$. Hye Seung Lee ${ }^{1}$

'Department of Pathology, Seoul National University Bundang Hospital, Seongnam; 2Department of Pathology, Seoul National University College of Medicine, Seoul; ${ }^{3}$ Department of Surgery, Seoul National University Bundang Hospital, Seongnam, Korea

Received: February 12, 2016

Revised: March 15, 2016

Accepted: March 18, 2016

Corresponding Author

Hye Seung Lee, PhD

Department of Pathology, Seoul National University

Bundang Hospital, 82 Gumi-ro 173beon-gil,

Bundang-gu, Seongnam 13620, Korea

Tel: +82-31-787-7714

Fax: $+82-31-787-4012$

E-mail: hye2@snu.ac.kr

\begin{abstract}
Background: The aim of this study was to determine the regional heterogeneity and clinicopathological significance of microRNA-21 (miR-21) in advanced colorectal cancer (CRC) patients with distant metastasis. Methods: miR-21 expression was investigated by using locked nucleic acidfluorescence in situ hybridization in the center and periphery of the primary cancer and in distant metastasis from 170 patients with advanced CRC. In addition, $\alpha$-smooth muscle actin and desmin were evaluated to identify cancer-associated fibroblasts (CAFs) by using immunohistochemistry. Results: The miR-21 signal was observed in the cancer stroma. The expression of miR-21 (a score of 1-4) in the center and periphery of the primary cancer and in distant metastasis was observed in specimens from 133 (78.2\%), 105 (61.8\%), and 91 (53.5\%) patients, respectively. miR-21 expression was heterogeneous in advanced CRC. Discordance between miR-21 expression in the center of the primary cancer and either the periphery of the primary cancer or distant metastasis was $31.7 \%$ or $44.7 \%$, respectively. miR-21 stromal expression in the periphery of the primary cancer was significantly associated with a better prognosis $(p=.004)$. miR-21 expression was significantly associated with CAFs in the center of the primary cancer $(p=.001)$ and distant metastases $(p=.041)$. Conclusions: miR-21 expression is observed in cancer stroma related to the CAF quantity and frequently presents regional heterogeneity in CRC. Our findings indicate that the role of miR-21 in predicting prognosis may be controversial but provide a new perspective of miR-21 level measurement in cancer specimens.
\end{abstract}

Key Words: Colorectal neoplasms; MicroRNA-21; Neoplasm metastasis; Genetic heterogeneity
MicroRNAs (miRNAs) are small (18-25 nucleotides), endogenous, non-coding RNAs that act as post-transcriptional modulators of all cellular processes, including proliferation, differentiation, and apoptosis. ${ }^{1}$ Alterations in miRNA expression are associated with the deregulation of oncogenes and tumor suppressor genes. ${ }^{2}$ The discovery that miRNA expression is deregulated in cancer suggests the potential of miRNA as biomarkers for cancer. ${ }^{3}$ Therefore, targeting miRNAs is regarded as a potential therapeutic strategy and may be a promising diagnostic tool.

MiRNA-21 (miR-21) is consistently upregulated in various cancers, including the colon, stomach, lung, and breast cancer. ${ }^{4-7}$ Previous studies proved that miR-21 expression correlates with carcinogenesis. ${ }^{8-11}$ Some studies indicated that miR-21 expression is closely associated with poor prognosis in various cancers. ${ }^{4,12,13}$ In colorectal cancer (CRC), miR-21 functions as an onco-miRNA due to its key roles in proliferation, invasion, and metastasis. ${ }^{10,14}$ Although the mechanisms underlying the regulation of miR-21 in $\mathrm{CRC}$ remain to be defined, miR-21 can promote tumorigenesis in CRC. Some studies reported that plasma miR-21 is a potential noninvasive biomarker for early detection and prognosis of CRC. ${ }^{15-17}$ Additionally, miR-21 expression is greatly increased in chemotherapy-resistant CRC cells. ${ }^{18,19}$ Recent reports suggest that increased expression of miR-21 predicts poor prognosis in patients with CRC. ${ }^{20-22}$ However, these results were restricted to patients with stage II CRC. ${ }^{21-24}$ Thus, the prognostic value of miR-21 alterations in patients with advanced CRC presenting metastases is controversial.

We previously evaluated cancer-associated fibroblasts (CAFs) in advanced CRC with synchronous or metachronous distant metastases. ${ }^{25}$ CAFs facilitate the communication between tumor cells and the tumor microenvironment. In addition, they regulate tumor invasion and metastasis. ${ }^{26,27}$ Interestingly, most studies reported that miR-21 was predominantly observed in CAFs, not in cancer cells. ${ }^{21,22,24}$ These findings point to a dynamic malignant 
role of CAFs through miR-21 expression in CRC. Nevertheless, the correlation between miR-21 and CAF status is yet to be demonstrated in CRC.

Recently, systemic chemotherapy and targeted therapy have been used in patients with advanced CRC, increasing patient survival. ${ }^{28}$ Despite advances in medicine, some patients with CRC respond poorly. ${ }^{29}$ Although the reasons for drug resistance are not fully understood, they may be related to the presence of tumor heterogeneity. ${ }^{30-32}$ Previous reports indicated that regional heterogeneity of miRNA expression is observed in various cancer types. ${ }^{33-35}$ Therefore, variation of miR-21 expression between the primary tumor and metastatic sites needs to be elucidated in patients with advanced CRC.

The aim of this study was to evaluate the clinical significance of miR-21 and analyze its heterogeneity in patients with advanced $C R C$ presenting metastases. Additionally, we analyzed the correlation between CAFs and miR-21 status.

\section{MATERIALS AND METHODS}

\section{Patients and samples}

To evaluate the clinicopathological significance and heterogeneity of miR-21 status, 170 patients with advanced CRC presenting synchronous or metachronous metastases who had undergone surgical resection at Seoul National University Bundang Hospital between May 2003 and December 2009 were enrolled. The clinicopathological characteristics were obtained from the patients' medical records and pathology reports. Follow-up information, including patient's outcome and the interval between the date of surgical resection and death was collected. Data from patients lost to follow-up or those who had died from causes other than CRC were censored.

\section{Ethical statement}

All samples were obtained from surgically resected tumors examined pathologically at the Department of Pathology, Seoul National University Bundang Hospital. All samples and medical record data were anonymized before use in this study and the participants did not provide written informed consent. The use of medical record data and tissue samples for this study was approved by the Institutional Review Board of Seoul National University Bundang Hospital (reference No. B-1109/136-302).

\section{Tissue array method}

Surgically resected primary CRC specimens were formalinfixed and paraffin-embedded (FFPE). For each case, representa- tive areas of the donor blocks were obtained and rearranged into new recipient blocks (Superbiochips Laboratories, Seoul, Korea). A single core was $2 \mathrm{~mm}$ in diameter and those containing > 20\% tumor cells were considered valid cores.

\section{miRNA in situ hybridization}

Tissue microarray slides were processed by using locked nucleic acid (LNA)-fluorescence in situ hybridization (FISH) oligonucleotide probes for miR-21 and U6, both labeled with fluorescein at the 5'-end, according to the protocol described in the preparation protocol. After deparaffinization, the slides were incubated with proteinase- $\mathrm{K}$ and endogenous peroxidase was blocked with $3 \% \mathrm{H}_{2} \mathrm{O}_{2}$. Next, the slides were incubated with hybridization mix containing $1 \mathrm{nM}$ LNA U6 snRNA probe (Exiqon, Vedbaek, Denmark) and $20 \mathrm{nM}$ doubled-DIG LNA miR-21 probe (Exiqon) in ThermoBrite (Abbott Laboratories, Abbott Park, IL, USA) for 1 hour at $50^{\circ} \mathrm{C}$. Next, the slides were incubated in blocking solution and antifluorescein-horseradish peroxidase antibody (1:125, PerkinElmer, Shelton, CT, USA) for 1 hour. The signals were then amplified using Tyramide Signal Amplification (TSA-plus FITC, 1:50, PerkinElmer) for 10 minutes at room temperature. After incubation, the slides were mounted directly with SlowFade Gold antifade reagent with DAPI (Invitrogen, Carlsbad, CA, USA). All steps beginning with hybridization were performed in the dark.

The experimental data were interpreted according to the instructions in the RNAscope FFPE Assay Kit (Advanced Cell Diagnostics, Hayward, CA, USA): no staining observed at $40 \times$ magnification (score of 0 ); difficult to see at $40 \times$ magnification (score of 1); difficult to see at $20 \times$ magnification, but detectable staining at $40 \times$ magnification (score of 2); difficult to see at $10 \times$ magnification, but detectable staining at $20 \times$ magnification (score of 3); and detectable staining at $10 \times$ magnification (score of 4). A score of 1-4 indicates miR-21 overexpression (Fig. 1).

\section{Immunohistochemistry}

Array slides were labeled by immunohistochemistry using antibodies for smooth muscle actin (SMA; 1:1,000, Neomarkers, Fremont, CA, USA) and desmin (1:300, Dako, Glostrup, Denmark) after a microwave antigen retrieval procedure, except for SMA. The staining procedures were carried out using the ultraView Universal DAB Kit (Ventana Medical Systems, Tucson, AZ, USA) and an automated stainer (BenchMark XT, Ventana Medical Systems), according to the manufacturer's instructions. 

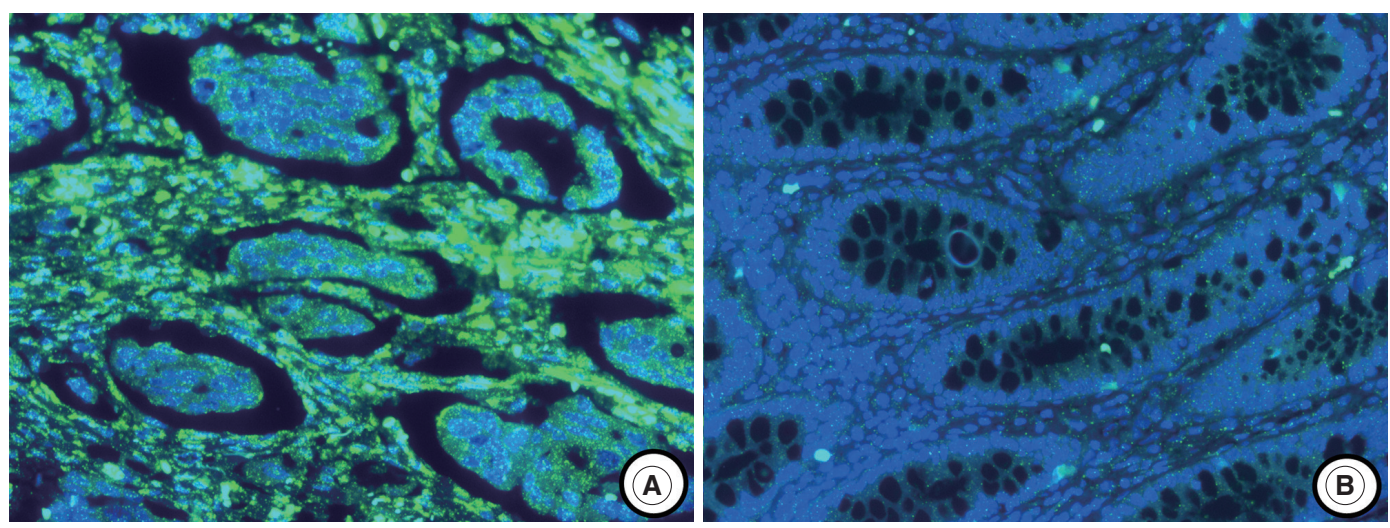

Fig. 1. MicroRNA-21 (miR-21) staining using locked nucleic acid-based in situ hybridization in colorectal cancer. (A) miR-21 expression is observed in cancer stromal tissue. (B) No miR-21 expression is observed in normal colonic mucosa.

Table 1. Heterogeneity of miR-21 expression with respect to tumor location in advanced CRC

\begin{tabular}{|c|c|c|c|c|}
\hline \multirow{2}{*}{\multicolumn{2}{|c|}{ miR-21 expression }} & \multicolumn{2}{|c|}{ Center } & \multirow{2}{*}{ Total } \\
\hline & & Negative & Positive & \\
\hline \multirow[t]{2}{*}{ Periphery } & Negative & $24(14.1)$ & $41(24.1)$ & $170(100)$ \\
\hline & Positive & $13(7.6)$ & $92(54.1)$ & \\
\hline \multirow[t]{2}{*}{ Distant metastasis } & Negative & $20(11.8)$ & $59(34.7)$ & $170(100)$ \\
\hline & Positive & $17(10.0)$ & 74 (43.5) & \\
\hline
\end{tabular}

Values are presented as number (\%). p-values are calculated by using chi-square test or Fisher exact test.

miR-21, microRNA-21; CRC, colorectal cancer.

\section{Calculation of CAFs using digital pathology}

CRC cells were considered as internal negative controls. Intestinal muscular layer or medium- to large-sized vessels were considered as internal positive controls for desmin and SMA. Samples showing inappropriate staining in internal negative or positive controls were considered non-informative and were excluded from the analysis. Slides were scanned using an Aperio ScanScopeH CS instrument (Aperio Technologies Inc., Vista, CA, USA) at $20 \times$ magnification. Because desmin-positive muscularis mucosa and propria are positive for SMA staining, the area of CAFs $\left(\mathrm{mm}^{2}\right)$ was calculated by subtracting the areas of desmin staining from that of SMA staining (SMA-desmin).

\section{Microsatellite instability}

Microsatellite instability (MSI) was assessed in 160 CRC cases with available tissue. MSI results were generated by comparing the allelic profiles of five microsatellite markers (BAT-26, BAT25, D5S346, D17S250, and D2S123) in the tumors and corresponding normal samples. Polymerase chain reaction products from the FFPE tissues were analyzed using an automated DNA sequencer (ABI 3731 Genetic Analyzer, Applied Biosystems, Foster City, CA, USA) according to the protocol.

\section{Statistical analyses}

The association between the clinicopathological features and miR-21 status was analyzed by using the chi-square or Fisher exact test, as appropriate. The correlation between miR-21 expression and CAFs was examined by using the Mann-Whitney test. The patients' survival was analyzed by using the KaplanMeier method and the log-rank test was used to determine if there were any significant differences between the survival curves. A p-value $<.05$ was considered statistically significant. All statistical analyses were performed using the SPSS statistics ver. 21 software (IBM Corp., Armonk, NY, USA).

\section{RESULTS}

\section{miR-21 stromal expression and regional heterogeneity in advanced CRC patients}

The miR-21 signal was predominantly observed in the stromal compartment of the cancer and the normal mucosa was negative for miR-21 (Fig. 1). The snRNA U6 signal was observed in the nucleus of all cell types. To evaluate the regional heterogeneity of miR-21 expression, we examined tissues from three sites, including the center and periphery of primary cancer as well as distant metastases for each patient with advanced CRC. In the center of primary tumors, a miR-21 FISH score of 0 was 
observed in $37(21.8 \%)$, a score of 1 in $16(9.4 \%)$, a score of 2 in $56(32.9 \%)$, a score of 3 in $46(27.1 \%)$, and a score of 4 in 15 $(8.8 \%)$ patients with CRC. In the periphery of primary tumors, a miR-21 FISH score of 0 was observed in $65(38.2 \%)$, a score of 1 in $14(8.2 \%)$, a score of 2 in $46(27.1 \%)$, a score of 3 in 44 (25.9\%), and a score of 4 in one (0.6\%) patient with CRC. Additionally, in distant metastatic tumors, a miR-21 FISH score of 0 was observed in 79 (46.5\%), a score of 1 in $13(7.6 \%)$, a score of 2 in $43(25.3 \%)$, a score of 3 in $32(18.8 \%)$, and a score of 4 in three (1.8\%) patients with CRC. miR-21 stromal expression (a score of 1-4) in the center and periphery of primary tumor as well as in distant metastasis was observed in $133(78.2 \%)$, 105 (61.8\%), and 91 (53.5\%) patients with CRC, respectively.

The heterogeneity of miR-21 status according to tumor location is shown in Table 1. Of the 170 cases, discordance between miR-21 expression in the center and periphery was noted in 54

Table 2. The association between clinicopathological parameters and expression of miR-21 in 170 advanced CRC patients with metastasis

\begin{tabular}{|c|c|c|c|c|c|c|c|c|c|c|}
\hline \multirow{3}{*}{ Variable } & \multirow{3}{*}{ Total } & \multirow{2}{*}{\multicolumn{2}{|c|}{$\begin{array}{c}\text { Center } \\
\text { miR-21 }\end{array}$}} & \multirow{3}{*}{$p$-value } & \multirow{2}{*}{\multicolumn{2}{|c|}{$\begin{array}{c}\text { Periphery } \\
\text { miR-21 }\end{array}$}} & \multirow{3}{*}{$p$-value } & \multirow{2}{*}{\multicolumn{2}{|c|}{$\begin{array}{c}\text { Metastasis } \\
\mathrm{miR}-21\end{array}$}} & \multirow{3}{*}{$p$-value } \\
\hline & & & & & & & & & & \\
\hline & & Negative & Positive & & Negative & Positive & & Negative & Positive & \\
\hline \multicolumn{11}{|l|}{ Age (yr) } \\
\hline Mean & 60.0 & 60.7 & 59.7 & .668 & 60.4 & 59.6 & .661 & 59.8 & 60.1 & .875 \\
\hline \multicolumn{11}{|l|}{ Sex } \\
\hline Male & 90 & $21(12.4)$ & $69(40.6)$ & .599 & 31 (18.2) & $59(34.7)$ & .281 & 36 (21.2) & 54 (31.8) & .073 \\
\hline Female & 80 & $16(9.4)$ & $64(37.6)$ & & $34(20.0)$ & $46(27.1)$ & & 43 (25.3) & 37 (21.8) & \\
\hline \multicolumn{11}{|l|}{ pT stage } \\
\hline $0-2$ & 103 & 23 (13.5) & $80(47.1)$ & .825 & 36 (21.2) & $67(39.4)$ & .275 & 45 (26.5) & 58 (34.1) & .367 \\
\hline $3-4$ & 67 & 14 (8.2) & $53(31.2)$ & & $29(17.1)$ & $38(22.4)$ & & 34 (20.0) & 33 (19.4) & \\
\hline \multicolumn{11}{|l|}{ Differentiation } \\
\hline$L G$ & 148 & 33 (19.4) & 115 (67.6) & .662 & $50(29.4)$ & $98(57.6)$ & .002 & 70 (41.2) & 78 (49.5) & .575 \\
\hline$H G$ & 22 & $4(2.4)$ & 18 (10.6) & & $15(8.8)$ & $7(4.1)$ & & $9(5.3)$ & $13(7.6)$ & \\
\hline Location of primary tumor & & & & .299 & & & .260 & & & .925 \\
\hline Right colon & 41 & $8(4.7)$ & $33(19.4)$ & & $20(11.8)$ & $21(12.4)$ & & $20(11.8)$ & $21(12.4)$ & \\
\hline Left colon & 69 & $12(7.1)$ & $57(33.5)$ & & $23(13.5)$ & $46(27.1)$ & & 31 (18.2) & $38(22.4)$ & \\
\hline Rectum & 60 & $17(10.0)$ & $43(25.3)$ & & $22(12.9)$ & $38(22.4)$ & & $28(16.5)$ & $32(18.8)$ & \\
\hline \multicolumn{11}{|l|}{ LN metastasis } \\
\hline Absent & 31 & $7(4.1)$ & $24(14.1)$ & .903 & $6(3.5)$ & $25(14.7)$ & .017 & $15(8.8)$ & $16(9.4)$ & .813 \\
\hline Present & 139 & $30(17.6)$ & $109(64.1)$ & & $59(34.7)$ & $80(47.1)$ & & $64(37.6)$ & $75(44.1)$ & \\
\hline \multicolumn{11}{|l|}{ Lymphatic invasion } \\
\hline Absent & 55 & $9(5.3)$ & $46(27.1)$ & .238 & $15(8.8)$ & $40(23.5)$ & .042 & $28(16.5)$ & 27 (15.9) & .422 \\
\hline Present & 115 & $28(16.5)$ & $87(51.2)$ & & $50(29.4)$ & $65(38.2)$ & & $51(30.0)$ & $64(37.6)$ & \\
\hline \multicolumn{11}{|l|}{ Perineural invasion } \\
\hline Absent & 83 & $19(11.2)$ & $64(37.6)$ & .728 & $29(17.1)$ & $54(31.8)$ & .388 & $41(24.1)$ & $42(24.7)$ & .455 \\
\hline Present & 87 & $18(10.6)$ & $69(40.6)$ & & $36(21.2)$ & $51(30.0)$ & & $38(22.4)$ & $49(28.8)$ & \\
\hline \multicolumn{11}{|l|}{ Venous invasion } \\
\hline Absent & 118 & $24(14.1)$ & $94(55.3)$ & .497 & $42(24.7)$ & $76(44.7)$ & .286 & $58(34.1)$ & 60 (35.3) & .291 \\
\hline Present & 52 & $13(7.6)$ & $39(22.9)$ & & $23(13.5)$ & $29(17.1)$ & & $21(12.4)$ & 31 (18.2) & \\
\hline \multicolumn{11}{|l|}{ Tumor border } \\
\hline Expanding & 12 & $4(2.4)$ & $8(4.7)$ & .314 & $4(2.4)$ & $8(4.7)$ & .717 & $6(3.5)$ & $6(3.5)$ & .799 \\
\hline Infiltrative & 158 & $33(19.4)$ & $125(73.5)$ & & $61(35.9)$ & $97(57.1)$ & & $73(42.9)$ & $85(50.0)$ & \\
\hline \multicolumn{11}{|l|}{ Distant metastasis } \\
\hline Synchronous & 110 & $23(13.5)$ & $87(51.2)$ & .714 & $45(26.5)$ & $65(38.2)$ & .331 & $52(30.6)$ & $58(34.1)$ & .776 \\
\hline Metachronous & 60 & $14(8.2)$ & $46(21.7)$ & & $20(11.8)$ & $40(23.5)$ & & 27 (15.9) & $33(19.4)$ & \\
\hline \multicolumn{11}{|c|}{ pTNM stage at initial diagnosis } \\
\hline |, || & 20 & $5(2.9)$ & $15(8.8)$ & .709 & $4(2.4)$ & $16(9.4)$ & .074 & $9(5.3)$ & $11(6.5)$ & .888 \\
\hline III, IV & 150 & 32 (18.8) & $118(69.4)$ & & $61(35.9)$ & $89(52.4)$ & & 70 (41.2) & $80(47.1)$ & \\
\hline \multicolumn{11}{|l|}{ MSI status } \\
\hline MSS/MSI-L & 157 & 35 (21.9) & $122(76.3)$ & .650 & 59 (36.9) & $98(61.3)$ & .304 & 74 (46.3) & 83 (51.9) & .069 \\
\hline MSI-H & 3 & $1(0.6)$ & $2(1.3)$ & & $2(1.3)$ & $1(0.6)$ & & $3(1.9)$ & 0 & \\
\hline
\end{tabular}

Values are presented as number (\%). p-values are calculated by using chi-square test or Fisher exact test.

miR-21, microRNA-21; CRC, colorectal cancer; T, tumor; LG, low grade; HG, high grade; LN, lymph node; MSI, microsatellite instability; MSS, microsatellite stable; MSI-L, microsatellite instability-low; MSI-H, microsatellite instability-high. 
cases $(31.7 \%)$. Discordance between the center and distant metastasis was detected in 76 cases $(44.7 \%)$. Thus, regional heterogeneity of miR-21 stromal expression was very common in patients with advanced CRC.

\section{Clinical implications of miR-21 stromal expression in advanced CRC patients}

Table 2 shows the relationships between miR-21 status and the clinicopathological parameters of patients with advanced CRC. In the periphery of primary tumor, miR-21 expression was correlated with less aggressive features, including histologic low grade differentiation $(\mathrm{p}=.002)$. In addition, lymphatic invasion and lymph node metastasis was frequently observed in patients with CRC negative for miR-21 ( $\mathrm{p}=.042$ and $\mathrm{p}=.017$, respectively). There was no statistically significant correlation between the clinicopathological factors and miR-21 expression in the center of the primary tumors and distant metastatic tumors $(\mathrm{p}>.05)$. The expression of miR-21 in distant metastasis was different according to metastatic site (Table 3). miR-21 stromal expression was more frequently observed in lung metastasis and peritoneal seeding $(\mathrm{p}=.046)$.

Table 3. Expression of miR-21 in distant metastasis according to metastatic site in 170 advanced CRC patients

\begin{tabular}{lrccc}
\hline \multirow{2}{*}{ Site of metastasis } & \multirow{2}{*}{ Total } & \multicolumn{2}{c}{ miR-21 in metastasis } & \\
\cline { 3 - 4 } & & Negative & Positive & \\
\hline Liver & 76 & $42(24.7)$ & $34(20.0)$ & .046 \\
Lung & 37 & $13(7.6)$ & $24(14.1)$ & \\
Seeding & 37 & $13(7.6)$ & $24(14.1)$ & \\
Distant nodes & 2 & 0 & $2(1.2)$ & \\
Ovary & 18 & $11(6.5)$ & $7(4.1)$ & \\
Total & 170 & 79 & 91 & \\
\hline
\end{tabular}

Values are presented as number (\%).

miR-21, microRNA-21; CRC, colorectal cancer.

\section{Prognostic significance of miR-21 stromal expression in advanced CRC patients}

All 170 patients with advanced CRC were successfully followed up for inclusion in the survival analysis (Fig. 2). The mean follow-up time was 42 months (range, 1 to 105 months) and 73 patients (42.9\%) died from cancer during the follow-up period. Kaplan-Meier analysis showed that miR-21 stromal expression in the periphery of primary tumors was significantly associated with a better prognosis $(\mathrm{p}=.004)$. There was no significant correlation between the patients' prognosis and miR-21 expression in the center of primary tumors and distant metastases $(\mathrm{p}=.925$ and $\mathrm{p}=.863$, respectively).

\section{Correlation between miR-21 stromal expression and CAF value}

The regional heterogeneous values for $\mathrm{CAF}$ in $\mathrm{CRC}$ are shown in our previous study. ${ }^{25}$ The area occupied by CAFs was the lowest in distant metastases (median, 0.91 ; interquartile range [IQR], 0.68 to 1.18 ) than any other sites (median, 1.12; IQR, 0.88 to 1.41 in the center of primary tumors and median, 1.22; IQR, 0.96 to 1.54 in the periphery of primary tumors). Mann-Whitney test showed that miR-21 expression was significantly associated with CAFs in the center of primary tumors $(\mathrm{p}=.001)$ and distant metastases $(\mathrm{p}=.041)$. In the periphery of primary tumors, miR-21 overexpression was not correlated with $\mathrm{CAFs}(\mathrm{p}=$ .102) (Fig. 3).

\section{DISCUSSION}

Many predictive and prognostic molecular markers have been suggested for CRC. However, they are not reliably accepted due to a lack of reproducibility and validation. Previous studies pro-
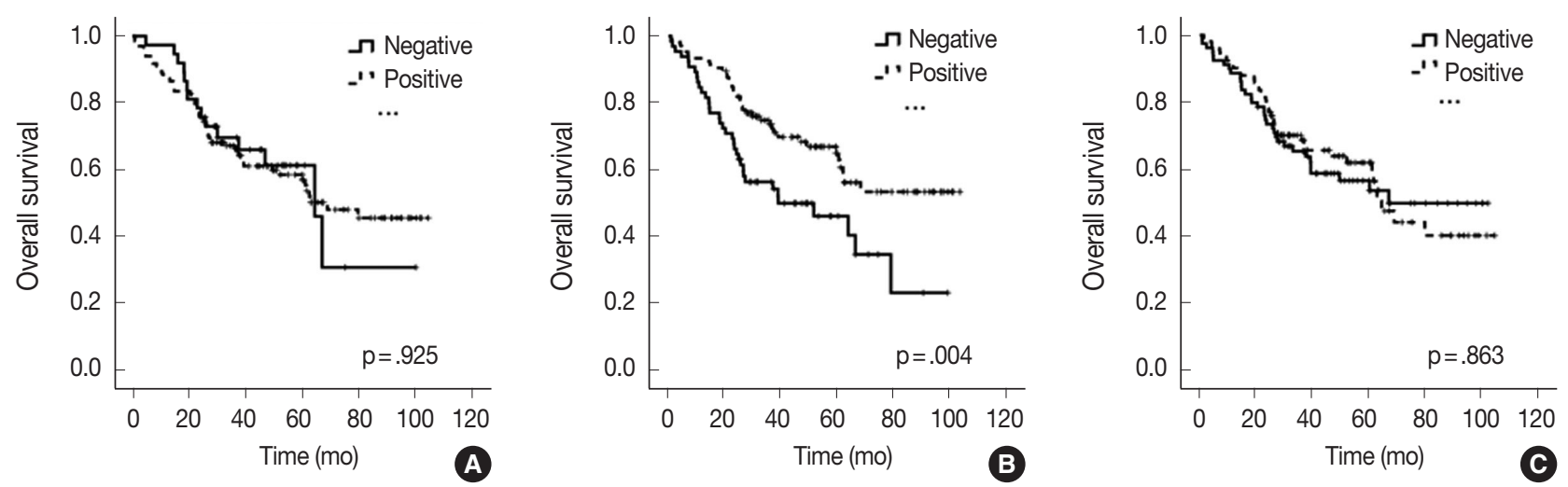

Fig. 2. Kaplan-Meier curves illustrating the overall survival of patients with advanced colorectal cancer in relation to microRNA-21 expression. (A) Center. (B) Periphery. (C) Distant metastasis. 

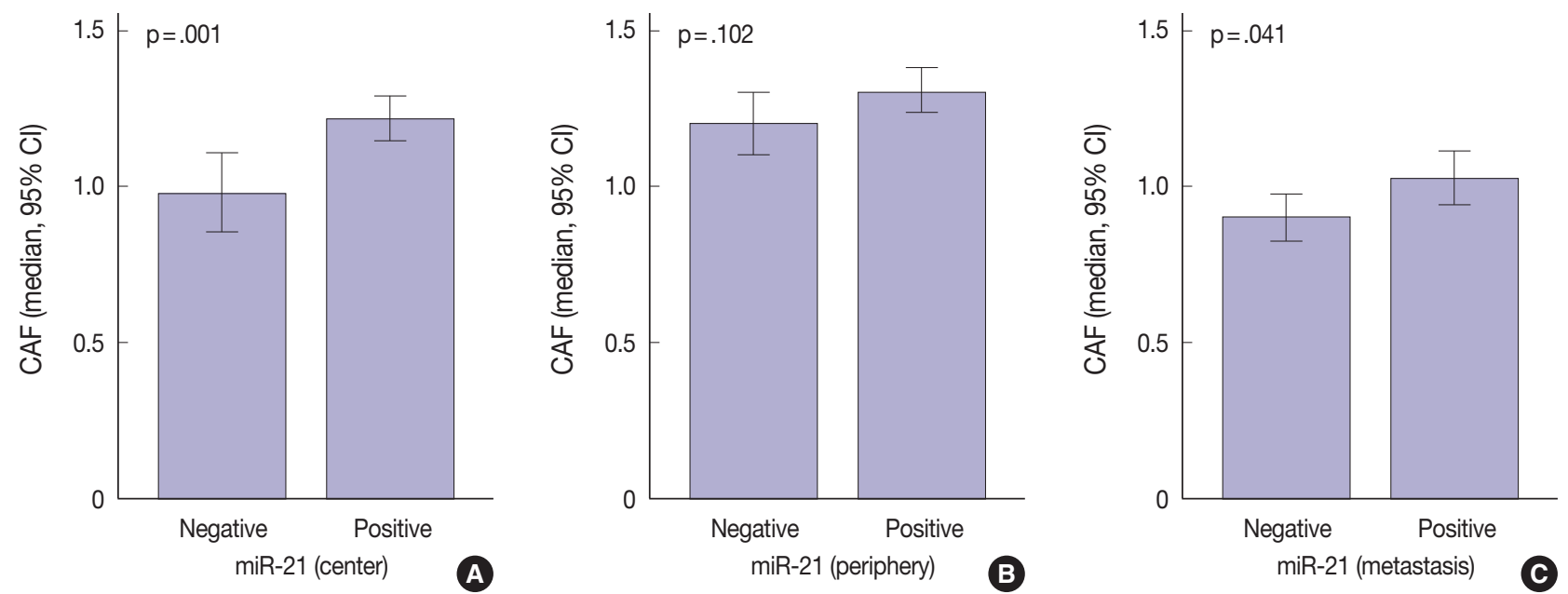

Fig. 3. Correlation between microRNA-21 (miR-21) expression and cancer associated fibroblasts (CAFs) by Mann-Whitney analysis. (A) Center. (B) Periphery. (C) Distant metastasis. Cl, confidence interval.

posed that high expression of miR-21 might predict poor survival in patients with CRC. ${ }^{20-24}$ However, elevated miR-21 level and poor prognosis correlated only in a subgroup of patients with stage II CRC. ${ }^{21-24}$ Interestingly, our results contradict those of previous miR-21 expression studies. In our study, miR-21 expression in the periphery of primary tumors was significantly associated with a better prognosis in patients with advanced stage CRC ( $\mathrm{p}=.004)$ (Fig. 2). Otherwise, there was no significant correlation between the prognosis and miR-21 expression in the center of primary tumors and in distant metastases $(\mathrm{p}=.925$ and $\mathrm{p}=.863$, respectively) (Fig. 2). This discrepancy may be explained by the fact that our cohort was largely comprised of patients with stage IV CRC (98 cases, 57.6\%). They received various personalized treatments and these might reflect the statistical difference.

Bullock et al..$^{36}$ recently demonstrated that stromal miR-21 expression induced a pro-metastatic mechanism of CRC via activation of matrix metalloproteinase-2. These data highlight the importance of miR-21 deregulation in CRC metastasis. Because all patients in our study presented with advanced CRC with metastasis, miR-21 may be expressed at higher levels than that observed in previous reports. In our study, miR-21 expression in the center and periphery of primary tumors and in distant metastasis was observed in $78.2 \%, 61.8 \%$, and $53.5 \%$ of the patients, respectively, whereas it was observed in $27.4 \%$ of the patients in another study. ${ }^{22}$ Our data suggest that the lack of miR21 expression in patients presenting with CRC with metastasis is associated with a rather poor prognosis and more frequent lymphatic invasion and lymph node metastasis (Table 2, Fig. 1), presumably because, in these patients, the metastatic mecha- nism is controlled by other regulatory pathways. Further studies are necessary to prove that other mechanisms induce CRC metastasis, independently of miR-21. The evaluation of the prognostic value of miR-21 expression in patients with advanced CRC presenting distant metastasis might be of little importance.

Previous studies in various cancers indicated that miR-21 localizes mainly in the cancer stroma and more particularly in the stromal fibroblast-like cells. ${ }^{21,22,24}$ This localization may be due to molecules secreted by cancer cells, which influence the microenvironment. ${ }^{37-39}$ Meanwhile, the mechanisms of regulation of miR-21 in CAFs remain unknown. Only few studies examined the correlation between the value of CAFs and miR-21 expression. ${ }^{40}$ Our study revealed that miR-21 expression was associated with CAF value in the center of primary tumors and distant metastases ( $\mathrm{p}=.001$ and $\mathrm{p}=.041$, respectively) (Fig. 3). As expected, the value of CAF was greater in stroma of CRC specimens presenting miR-21 expression. Therefore, we should be careful when evaluating stromal miR-21 expression. In most previous studies, miR-21 expression was evaluated quantitatively by image analysis. ${ }^{21,24,36}$ This method may mislead us when distinguishing miR-21 high expression from a simple increase in the number of CAFs. Thus, accurate observation should be performed to distinguish miR-21 expression from CAF increase.

To clarify miR-21 expression in CRC, being aware of the heterogeneity in miR-21 expression level is crucial because regional heterogeneity can lead to sampling bias. In recent studies, intra-tumor heterogeneity of various miRNAs was detected in colorectal, pancreatic, and breast cancer. ${ }^{34,35,41}$ Our study indicates miR-21 regional heterogeneity, which constitutes approximately $40 \%$ of the total cohort (Table 1). Thus, a reliable assessment 
of CRC miR-21 expression may include sampling of the primary tumor in several locations and metastasis.

In conclusion, we demonstrated that miR-21 is expressed in advanced CRC and that this upregulation is mainly confined to the cancer stroma. Our FISH data indicated that miR-21 expression is related to CAF value in the center of primary tumors and distant metastasis. We determined that miR-21 expression frequently presents regional heterogeneity in CRC. Such data could lead to the new perspective of miR-21 level measurement. The evaluation of miR-21 expression for the prediction of survival in patient with advanced CRC is a matter of debate.

\section{Conflicts of Interest}

No potential conflict of interest relevant to this article was reported.

\section{Acknowledgments}

This research was supported by a grant of the Korea Health Technology R\&D Project through the Korea Health Industry Development Institute (KHIDI), funded by the Ministry of Health \& Welfare, Republic of Korea (grant number: HI14C1813, https://www.khidi.or.kr/eps).

\section{REFERENCES}

1. Carrington JC, Ambros V. Role of microRNAs in plant and animal development. Science 2003; 301: 336-8.

2. Zhang B, Pan X, Cobb GP, Anderson TA. microRNAs as oncogenes and tumor suppressors. Dev Biol 2007; 302: 1-12.

3. Kwak PB, Iwasaki S, Tomari Y. The microRNA pathway and cancer. Cancer Sci 2010; 101: 2309-15.

4. Chan SH, Wu CW, Li AF, Chi CW, Lin WC. miR-21 microRNA expression in human gastric carcinomas and its clinical association. Anticancer Res 2008; 28: 907-11.

5. Gabriely G, Wurdinger T, Kesari S, et al. MicroRNA 21 promotes glioma invasion by targeting matrix metalloproteinase regulators. Mol Cell Biol 2008; 28: 5369-80.

6. Qian B, Katsaros D, Lu L, et al. High miR-21 expression in breast cancer associated with poor disease-free survival in early stage disease and high TGF-beta1. Breast Cancer Res Treat 2009; 117: 131-40.

7. Markou A, Tsaroucha EG, Kaklamanis L, Fotinou M, Georgoulias V, Lianidou ES. Prognostic value of mature microRNA-21 and microRNA-205 overexpression in non-small cell lung cancer by quantitative real-time RT-PCR. Clin Chem 2008; 54: 1696-704.

8. Zhang A, Liu Y, Shen Y, Xu Y, Li X. miR-21 modulates cell apopto- sis by targeting multiple genes in renal cell carcinoma. Urology 2011; 78: 474.

9. Hiyoshi Y, Kamohara H, Karashima R, et al. MicroRNA-21 regulates the proliferation and invasion in esophageal squamous cell carcinoma. Clin Cancer Res 2009; 15: 1915-22.

10. Asangani IA, Rasheed SA, Nikolova DA, et al. MicroRNA-21 (miR21) post-transcriptionally downregulates tumor suppressor Pdcd4 and stimulates invasion, intravasation and metastasis in colorectal cancer. Oncogene 2008; 27: 2128-36.

11. Lu Z, Liu M, Stribinskis V, et al. MicroRNA-21 promotes cell transformation by targeting the programmed cell death 4 gene. Oncogene 2008; 27: 4373-9.

12. Mathé EA, Nguyen GH, Bowman ED, et al. MicroRNA expression in squamous cell carcinoma and adenocarcinoma of the esophagus: associations with survival. Clin Cancer Res 2009; 15: 6192-200.

13. Yan LX, Huang XF, Shao Q, et al. MicroRNA miR-21 overexpression in human breast cancer is associated with advanced clinical stage, lymph node metastasis and patient poor prognosis. RNA 2008; 14: 2348-60.

14. Yu Y, Nangia-Makker P, Farhana L, Rajendra SG, Levi E, Majumdar AP. miR-21 and miR-145 cooperation in regulation of colon cancer stem cells. Mol Cancer 2015; 14: 98.

15. Kanaan Z, Rai SN, Eichenberger MR, et al. Plasma miR-21: a potential diagnostic marker of colorectal cancer. Ann Surg 2012; 256: 544-51.

16. Ng EK, Chong WW, Jin $\mathrm{H}$, et al. Differential expression of microRNAs in plasma of patients with colorectal cancer: a potential marker for colorectal cancer screening. Gut 2009; 58: 1375-81.

17. Huang Z, Huang D, Ni S, Peng Z, Sheng W, Du X. Plasma microRNAs are promising novel biomarkers for early detection of colorectal cancer. Int J Cancer 2010; 127: 118-26.

18. Yu Y, Sarkar FH, Majumdar AP. Down-regulation of miR-21 induces differentiation of chemoresistant colon cancer cells and enhances susceptibility to therapeutic regimens. Transl Oncol 2013; 6: 180-6.

19. Valeri N, Gasparini P, Braconi C, et al. MicroRNA-21 induces resistance to 5-fluorouracil by down-regulating human DNA MutS homolog 2 (hMSH2). Proc Natl Acad Sci U S A 2010; 107: 21098-103.

20. Xia X, Yang B, Zhai X, et al. Prognostic role of microRNA-21 in colorectal cancer: a meta-analysis. PLoS One 2013; 8: e80426.

21. Kjaer-Frifeldt S, Hansen TF, Nielsen BS, et al. The prognostic importance of miR-21 in stage II colon cancer: a population-based study. Br J Cancer 2012; 107: 1169-74.

22. Kang WK, Lee JK, Oh ST, Lee SH, Jung CK. Stromal expression of miR-21 in T3-4a colorectal cancer is an independent predictor of early tumor relapse. BMC Gastroenterol 2015; 15: 2.

23. Zhang JX, Song W, Chen ZH, et al. Prognostic and predictive value of a microRNA signature in stage II colon cancer: a microRNA ex- 
pression analysis. Lancet Oncol 2013; 14: 1295-306.

24. Nielsen BS, Jørgensen S, Fog JU, et al. High levels of microRNA-21 in the stroma of colorectal cancers predict short disease-free survival in stage II colon cancer patients. Clin Exp Metastasis 2011; 28: 27-38.

25. Kwak Y, Lee HE, Kim WH, Kim DW, Kang SB, Lee HS. The clinical implication of cancer-associated microvasculature and fibroblast in advanced colorectal cancer patients with synchronous or metachronous metastases. PLoS One 2014; 9: e91811.

26. Tsujino T, Seshimo I, Yamamoto $H$, et al. Stromal myofibroblasts predict disease recurrence for colorectal cancer. Clin Cancer Res 2007; 13: 2082-90.

27. Trimboli AJ, Cantemir-Stone CZ, Li F, et al. Pten in stromal fibroblasts suppresses mammary epithelial tumours. Nature 2009; 461: 1084-91.

28. Knijn N, Tol J, Punt CJ. Current issues in the targeted therapy of advanced colorectal cancer. Discov Med 2010; 9: 328-36.

29. O'Connell MJ, Campbell ME, Goldberg RM, et al. Survival following recurrence in stage II and III colon cancer: findings from the ACCENT data set. J Clin Oncol 2008; 26: 2336-41.

30. Albanese I, Scibetta AG, Migliavacca M, et al. Heterogeneity within and between primary colorectal carcinomas and matched metastases as revealed by analysis of Ki-ras and p53 mutations. Biochem Biophys Res Commun 2004; 325: 784-91.

31. Park JH, Han SW, Oh DY, et al. Analysis of KRAS, BRAF, PTEN, IGF1R, EGFR intron 1 CA status in both primary tumors and paired metastases in determining benefit from cetuximab therapy in colon cancer. Cancer Chemother Pharmacol 2011; 68: 1045-55.

32. Baldus SE, Schaefer KL, Engers R, Hartleb D, Stoecklein NH, Gabbert HE. Prevalence and heterogeneity of KRAS, BRAF, and PIK-
3CA mutations in primary colorectal adenocarcinomas and their corresponding metastases. Clin Cancer Res 2010; 16: 790-9.

33. Villegas-Ruiz V, Juárez-Méndez S, Pérez-González OA, et al. Heterogeneity of microRNAs expression in cervical cancer cells: overexpression of miR-196a. Int J Clin Exp Pathol 2014; 7: 1389-401.

34. Jepsen RK, Novotny GW, Klarskov LL, Christensen IJ, Riis LB, Høgdall E. Intra-tumor heterogeneity of microRNA-92a, microRNA-375 and microRNA-424 in colorectal cancer. Exp Mol Pathol 2016; 100: 125-31.

35. Raychaudhuri M, Schuster T, Buchner T, et al. Intratumoral heterogeneity of microRNA expression in breast cancer. J Mol Diagn 2012; 14: 376-84.

36. Bullock MD, Pickard KM, Nielsen BS, et al. Pleiotropic actions of miR-21 highlight the critical role of deregulated stromal microRNAs during colorectal cancer progression. Cell Death Dis 2013; 4: e684.

37. Yamamichi N, Shimomura $\mathrm{R}$, Inada $\mathrm{K}$, et al. Locked nucleic acid in situ hybridization analysis of miR-21 expression during colorectal cancer development. Clin Cancer Res 2009; 15: 4009-16.

38. Shenouda SK, Alahari SK. MicroRNA function in cancer: oncogene or a tumor suppressor? Cancer Metastasis Rev 2009; 28: 369-78.

39. Fassan M, Pizzi M, Giacomelli L, et al. PDCD4 nuclear loss inversely correlates with miR-21 levels in colon carcinogenesis. Virchows Arch 2011; 458: 413-9.

40. Aprelikova O, Green JE. MicroRNA regulation in cancer-associated fibroblasts. Cancer Immunol Immunother 2012; 61: 231-7.

41. Lou E, Subramanian S, Steer CJ. Pancreatic cancer: modulation of KRAS, microRNAs, and intercellular communication in the setting of tumor heterogeneity. Pancreas 2013; 42: 1218-26. 